\title{
Magnetic Nulls and Super-radial Expansion in the Solar Corona
}

\author{
Sarah E. Gibson ${ }^{1}$, Kevin Dalmasse ${ }^{1}$, Laurel A. Rachmeler ${ }^{2}$, Marc L. De Rosa ${ }^{3}$, Steven Tomczyk ${ }^{1}$, \\ Giuliana de Toma ${ }^{1}$, Joan Burkepile ${ }^{1}$, and Michael Galloy ${ }^{1}$ \\ ${ }^{1}$ National Center for Atmospheric Research, 3080 Center Green Drive, Boulder, CO 80301, USA; sgibson@ucar.edu \\ ${ }^{2}$ NASA Marshall Space Flight Center, Huntsville, AL 35811, USA \\ ${ }^{3}$ Lockheed Martin Solar and Astrophysics Laboratory, 3251 Hanover Street B/252, Palo Alto, CA 94304, USA \\ Received 2017 March 7; revised 2017 April 24; accepted 2017 April 24; published 2017 May 8
}

\begin{abstract}
Magnetic fields in the Sun's outer atmosphere-the corona-control both solar-wind acceleration and the dynamics of solar eruptions. We present the first clear observational evidence of coronal magnetic nulls in off-limb linearly polarized observations of pseudostreamers, taken by the Coronal Multichannel Polarimeter (CoMP) telescope. These nulls represent regions where magnetic reconnection is likely to act as a catalyst for solar activity. CoMP linear-polarization observations also provide an independent, coronal proxy for magnetic expansion into the solar wind, a quantity often used to parameterize and predict the solar wind speed at Earth. We introduce a new method for explicitly calculating expansion factors from CoMP coronal linear-polarization observations, which does not require photospheric extrapolations. We conclude that linearly polarized light is a powerful new diagnostic of critical coronal magnetic topologies and the expanding magnetic flux tubes that channel the solar wind.
\end{abstract}

Key words: solar wind - Sun: corona - Sun: magnetic fields

\section{Background}

Measuring the strength and direction of the solar coronal magnetic field is a fundamental requirement for understanding solar activity and evolution, but remains a significant challenge. When simplifying assumptions are used, such as the currentfree or potential limit to the magnetic field in the corona, observations of the magnetic field at the solar surface may be used to approximate the coronal field. Such photospheric extrapolations-e.g., the potential-field-source-surface (PFSS) model-provide a good first-order characterization of the global coronal and interplanetary magnetic field (Newkirk \& Altschuler 1969; Schatten et al. 1969). However, this model ignores currents, removing the component of the magnetic energy that drives solar eruptions. Another possibility is to use the full vector of the magnetic field at the photospheric boundary and solve a nonlinear force-free field (NLFFF) problem (under the assumption that magnetic forces dominate in the highly conductive corona). However, issues such as a lack of magnetic dominance at the photosphere lead to inconsistencies and non-uniqueness of NLFFF models (De Rosa et al. 2009). The observed photospheric boundary on its own is therefore not sufficient to model the coronal magnetic field, motivating the explicit incorporation of coronal observations into magnetic models.

The Coronal Multichannel Polarimeter (CoMP; Tomczyk et al. 2008) obtains daily observations of coronal emission-line polarization in the solar atmosphere, providing unique constraints on coronal magnetic models. Polarimetry has been used to determine magnetic fields at the solar surface since Hale's observations in 1908. More recently, routine measurements of magnetism in its lower atmosphere, or chromosphere, have become possible (Ariste 2002; Harvey 2006). At higher coronal altitudes, emission-line measurements of circular polarization - sensitive to line of sight (LOS) oriented magnetic-field strength—have been rare (Harvey 1969; Lin \& Casini 2000; Lin et al. 2004; Tomczyk et al. 2008). Obtaining routine circular-polarization measurements at the level of
1 Gauss in 15 minutes generally requires a larger-aperture telescope than the $20 \mathrm{~cm}$ CoMP (Tomczyk et al. 2016). On the other hand, linear polarization, which is approximately 100 times brighter than circular polarization, is measured by CoMP on a daily basis. As we now demonstrate, linear-polarization observations are ideally suited for probing certain topological and expansion properties of the coronal magnetic field.

\section{Linear-polarization Diagnostics: Magnitude and Azimuth}

The Fe XIII coronal emission line at $1074.7 \mathrm{~nm}$ is linearly polarized when unpolarized light emitted from lower layers of the solar atmosphere undergoes scattering in the corona. However, magnetic fields in the corona partially depolarize it, a process known as the Hanle effect (Sahal-Bréchot 1977; Querfeld \& Smartt 1984; Arnaud \& Newkirk 1987). In the corona, at this wavelength, the depolarization of the scattered light preserves information about the direction of the magnetic field, but not its strength. In particular, if the local magnetic vector has an angle $\vartheta_{B}$ relative to the solar radius vector $(\hat{r})$ that is less than a critical "van Vleck" angle (van Vleck 1925), its projection into the plane of sky (POS) lies parallel to the observed linear-polarization vector. However, if $\vartheta_{B}$ is greater than this critical angle, then the direction of the linearpolarization vector (known as the azimuth) is rotated $90^{\circ}$. The critical van Vleck angle, $\vartheta_{B}=54^{\circ} .74$, arises because of an atomic-alignment dependence of the form $3 \cos ^{2} \vartheta_{B}=1$; at this critical angle, the fraction of linearly polarized light (linearpolarization magnitude) is zero.

CoMP observations and forward modeling have demonstrated that, in certain magnetic topologies, the loci of van Vleck angle crossings can be picked out of linear-polarization magnitude observations as coherent and elongated dark features. The corona is optically thin in the Fe XIII line, and observations are intensity-weighted along the LOS, so multiple bright structures of varying magnetic orientations can in general contribute to the linear polarization observed by 
a)

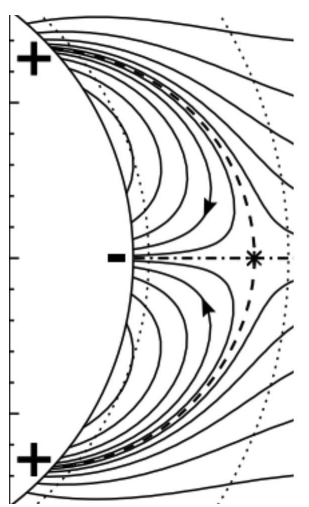

b)

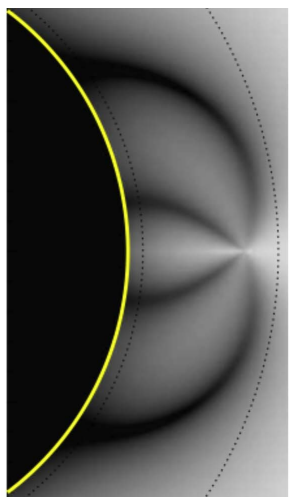

C)

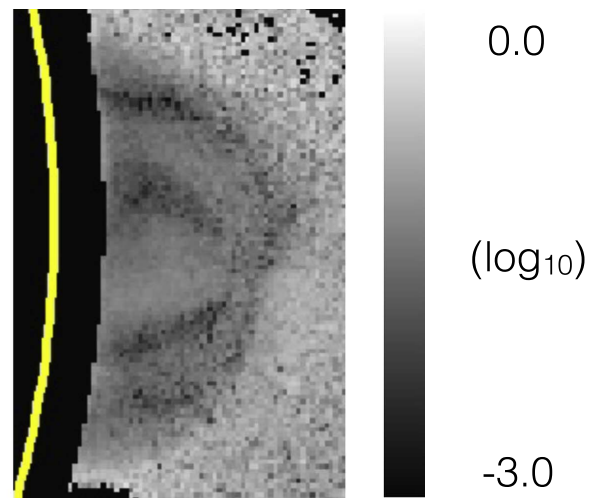

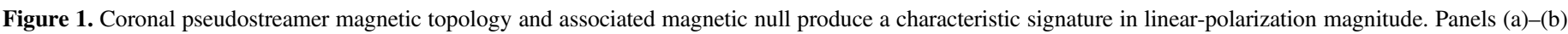

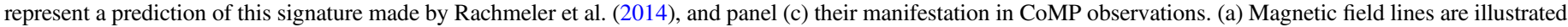

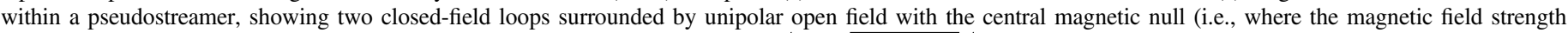

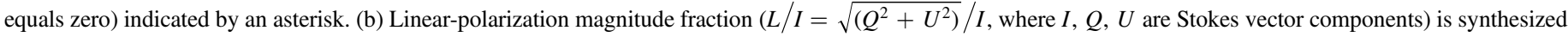

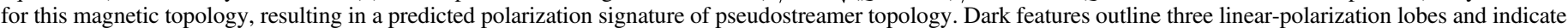

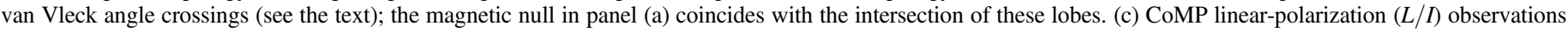

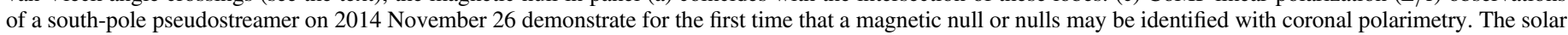
photosphere is indicated by the yellow curves in panels (b) and (c).

CoMP. Thus, the presence of distinct van Vleck loci implies a degree of isolation and/or extension along the LOS of the magnetic structure. Under such conditions, these loci have proved useful for identifying coronal topologies. For example, the linear-polarization signatures expected from forward modeling cylindrical versus spheromak magnetic flux ropes are distinct (Rachmeler et al. 2013). CoMP observations have provided one example of a possible spheromak (Dove et al. 2011); far more examples have been found of coronal prominence cavities (Gibson 2015) that, for many sizes and shapes, possess "lagomorphic" (rabbit-headed) signatures in linear-polarization magnitude, consistent with a cylindrical magnetic flux-rope topology (Ba̧k-Stęślicka et al. 2013, 2014).

\section{Coronal Magnetic Nulls from Linear-polarization Magnitude}

Coronal pseudostreamers and associated magnetic nulls are likely locations for a restructuring of the magnetic field to be triggered, potentially leading to coronal mass ejections (Antiochos et al. 1999; Török et al. 2011; Lynch \& Edmondson 2013). Pseudostreamers consist of multipolar closed field surrounded by unipolar open magnetic field (Hundhausen 1972; Zhao \& Webb 2003; Wang et al. 2007). They are distinguished from so-called "helmet" (or bipolar) streamers, which are surrounded by open field of opposite polarities. Magnetic null points are expected in pseudostreamer topologies, as shown in Figure 1(a). Although pseudostreamers are three-dimensional magnetic structures, this two-dimensional idealization is a reasonable representation of the middle portion of a pseudostreamer that is oriented along an observer's LOS. As discussed above and further demonstrated below, such an optimal orientation is in fact a necessary selection factor for obtaining clear linear polarization signatures. We will refer to magnetic nulls throughout this Letter; however, we note that this effectively two-dimensional interpretation does not distinguish between a true, three-dimensional magnetic null point and topologically related structures such as a superposition of a line of nulls, a magnetic separator, or a hyperbolic flux tube (see, e.g., Titov et al. 2002, 2011; Aulanier et al. 2005; Parnell et al. 2010).

Forward models of coronal pseudostreamer magnetic configurations have predicted a characteristic signature of three linear-polarization lobes (bright regions outlined by van Vleck dark features; see Figure 1(b)), which come to a point at the magnetic null (Rachmeler et al. 2014). In that work, CoMP data were presented that were consistent with the predicted pseudostreamer configuration, but that did not extend high enough to include the magnetic null. We have now observed a pseudostreamer with CoMP data that unequivocally matches the forward-modeled prediction of the pseudostreamer topology and explicitly contains the signature of the converging lobes indicative of magnetic null(s) at their top (Figure 1(c)).

Figures 2 and 3 present another example of a pseudostreamer and directly compare CoMP data to PFSS model predictions. Synthetic data are generated using the "pfss" and "FORWARD" (Gibson et al. 2016) packages of SolarSoft IDL (Freeland \& Handy 1998). Plasma weighting along the LOS is achieved by defining density radial profiles consistent with hydrostatic equilibrium: closed-field regions are assigned profiles that match coronal streamer observations (Gibson et al. 1999), while open-field regions are given profiles to match coronal hole observations (Guhathakurta et al. 1999).

Note that although more asymmetric than the pseudostreamer of Figure 1, the lobes of this pseudostreamer are apparent in linear polarization for both CoMP (Figure 2(c)) and forwardmodeled PFSS (Figure 3(c)). PFSS magnetic field plots show this topology even more clearly (Figures 3(a)-(b)). However, a visual inspection of the CoMP observations indicate a higher altitude null than the PFSS model possesses. The location of this CoMP null coincides with the base of the sharp linear feature extending above the pseudostreamer dome and up into the pseudostreamer stalk, as seen by the Solar Dynamics Observatory Atmospheric Imaging Assembly (AIA) images (Figures 2(a)-(b)). We note that this feature is somewhat to the left of the center of the dome, but also that the CoMP linear polarization feature is similarly asymmetric. The PFSS model also does not fully capture the bulging nature of the northward 

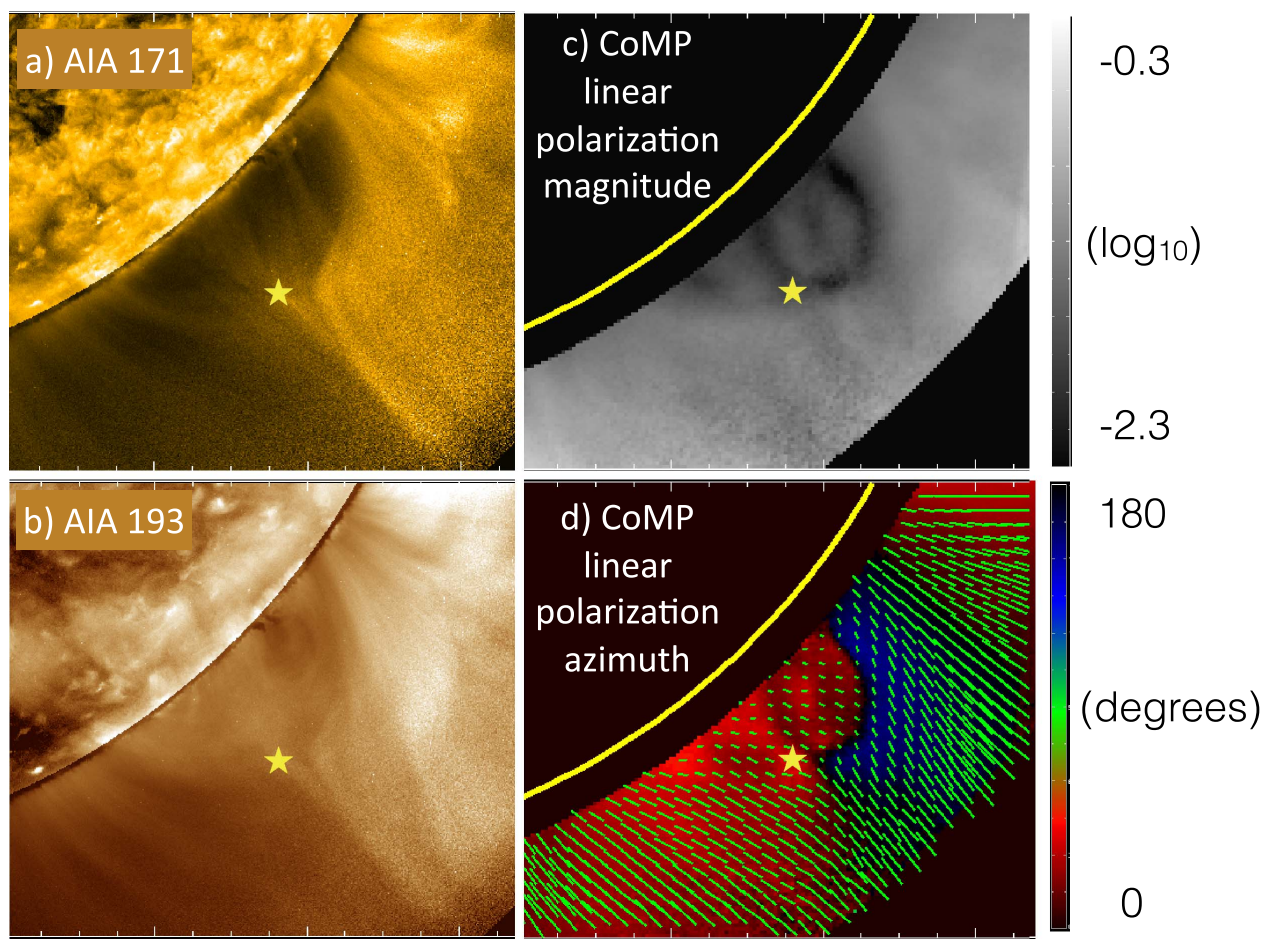

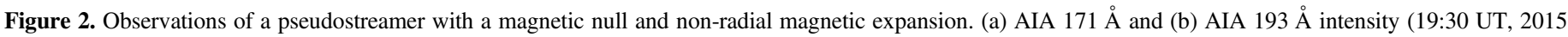

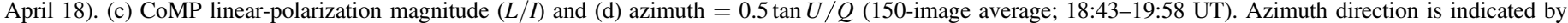

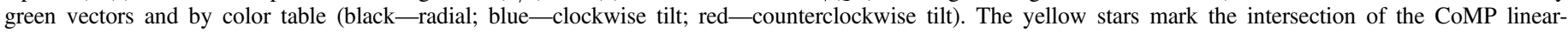
polarization magnitude lobes determined by eye from panel (c). The solar photosphere is indicated by the yellow curves in panels (c) and (d).

lobe seen in the CoMP images. The AIA $193 \AA$ image (Figure 2(b) shows the presence of a prominence cavity within this lobe, so it is possible the expanded lobe is a consequence of currents associated with a flux rope that are missed by the (current-free) PFSS model.

\section{Coronal Non-radial Magnetic Expansion from Linear-polarization Azimuth}

Another important coronal property is the height-dependent expansion of open magnetic field surrounding closed-field structures such as helmet streamers and pseudostreamers. The degree of super-radial expansion of open-field regions is of particular interest because it has been shown to be inversely correlated to solar-wind speed (Wang \& Sheeley 1990). A magnetic expansion factor, $f$ (see the Appendix, Equation (2); also Figure 3(b)), may be calculated from measurements at the solar photosphere along with, e.g., PFSS model evaluations at higher altitudes. This is then a standard input into empirical models that predict solar-wind speed at the Earth (e.g., Arge \& Pizzo 2000).

CoMP linear-polarization observations present us with a new resource for probing magnetic flux-tube expansion as a function of height in the low corona, independent of model extrapolations. Figures 2(d) and 3(d) illustrate that van Vleck crossings manifest not only as dark features in linearpolarization magnitude, but also as discontinuities in linearpolarization azimuth, so that diverging versus converging magnetic fields have distinct appearances. Thus, azimuths crossing the van Vleck angle-for example within the closedfield loops that make up the pseudostreamer-result in sharp jumps from red to blue. In contrast, the diverging magnetic fields to the north-associated with magnetic flux tubes open to the solar wind-have a gradual transition from blue to red and no van Vleck crossing. However, the sharp jump at the van Vleck angle may be blurred if, for example, the locus of these angles do not superpose along the LOS; this appears to be the case for the southern van Vleck crossings for both the CoMP observations and the synthetic PFSS data.

A visual comparison of the CoMP-observed azimuth angle (Figure 2(d)) and the PFSS forward-modeled azimuth angle (Figure 3(d)) suggests that the PFSS extrapolation underestimates the super-radiality of magnetic expansion to the north of the pseudostreamer. To further investigate this, we create maps of an expansion-factor proxy from both the CoMP and the synthetic PFSS data (Figure 4). Using a predictor-corrector Euler algorithm and bilinear interpolation, we integrate polarization field lines following the azimuth down to the lower CoMP occulter. A linear-polarization expansion factor (LPF) is then determined from the local polarization field line deviation (see the Appendix). We find that the CoMP data in this region indicates a more super-radial expansion than the PFSS model predicts. We note that this technique relies on an interpretation of the azimuth as an effectively two-dimensional POS field which only holds true in regions that are not heavily impacted by LOS effects, and that have magnetic field orientation tilted less than the van Vleck angle from radial. The clear identification and localization of the van Vleck inversion to the bottom left (see also Figure 3) gives us confidence that both conditions are met for the diverging, open magnetic field shown in this figure and used to calculate the LPF.

\section{Discussion}

We have shown how CoMP linear-polarization observations may be used to quantify both magnetic topology (height of 


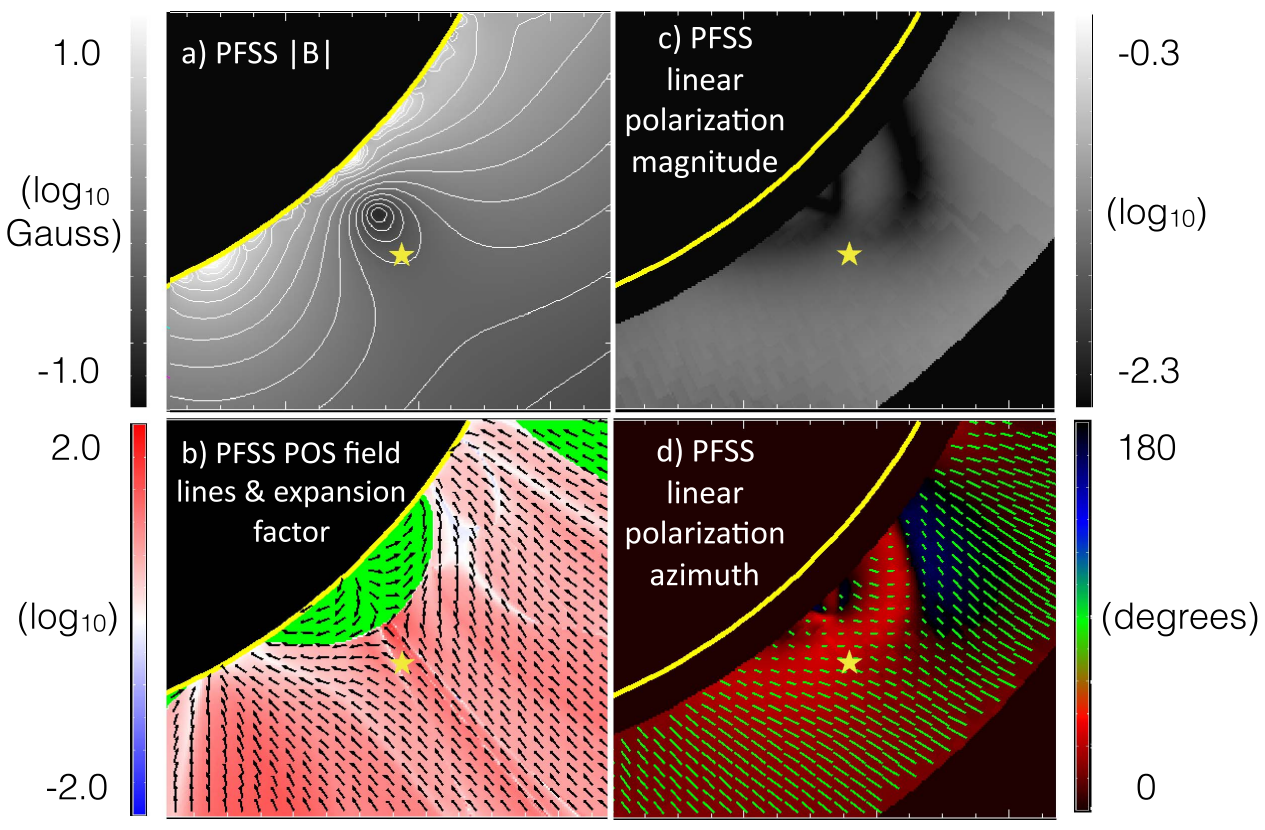

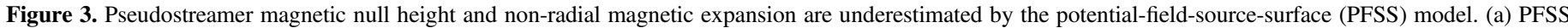

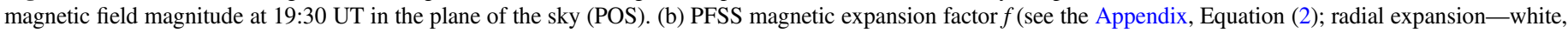

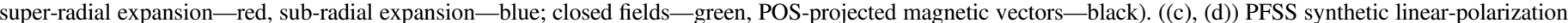

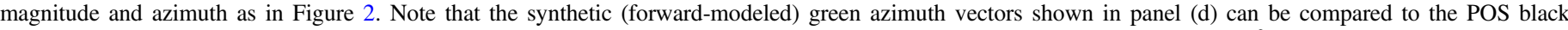

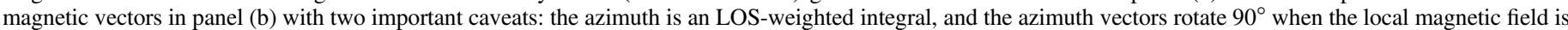

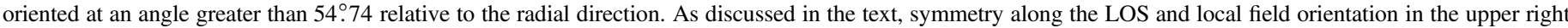

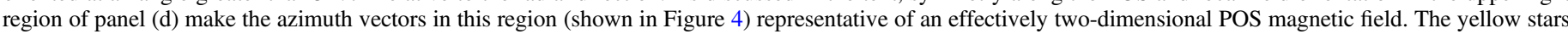

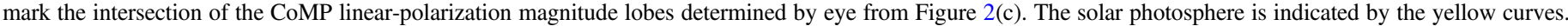

magnetic nulls) and super-radial expansion (LPF as a function of height and latitude). The detection of magnetic nulls provides a new way to identify critical points in the coronal field that facilitate solar eruptions. The linear-polarization expansion factor (LPF) proxy provides a new means for validating PFSS (and other) model predictions.

It has been pointed out that pseudostreamer-sourced solar wind may be inconsistent with the empirical relationship between PFSS-model-derived expansion factor and wind speed (Riley et al. 2011). In particular, the authors observed solar wind associated with a pseudostreamer to be slow, in contrast to the fast wind predicted by low PFSS-derived expansion factors. Wang et al. (2012) have also pointed out that the null-point height affects magnetic expansion, and that nonmonotonically expanding flux tubes may result in an overestimate of solar wind speed. The CoMP observations we have presented demonstrate how discrepancies between observations and the empirically predicted solar wind speed could at least partially arise from an underestimate of magnetic expansion and null-point height by the PFSS model due to intrinsic limitations of photospheric extrapolations. It is also possible that reconnections at the openclosed magnetic boundary rather than flux-tube expansion is the dominant physical process controlling the solar wind speed associated with pseudostreamers, as has been argued in other models (e.g., Antiochos et al. 2011; Del Zanna et al. 2011). These models too require accurate determination of coronal magnetic topology, in particular magnetic null point location. In conclusion, coronal linear-polarization measurements represent a valuable new type of data for validating and developing nextgeneration coronal and solar-wind acceleration models.

NCAR is supported by the National Science Foundation. S. E.G., K.D., G.d.T., and S.T. acknowledge support from the Air
Force Office of Space Research, FA9550-15-1-0030. K.D. acknowledges funding from the Computational and Information Systems Laboratory, the High Altitude Observatory, and the Advanced Study Program. AIA data are courtesy of NASA/SDO and the AIA, EVE, and HMI science teams. We acknowledge helpful discussions with Anna Malanushenko, Ed Deluca, Antonia Savcheva, and Scott McIntosh.

\section{Appendix}

\section{Computing a Linear-polarization Expansion Factor (LPF)}

\section{A.1. Background}

A magnetic expansion factor measures the degree to which the cross sections of open magnetic flux tubes expand (or contract) in the solar corona as compared with their cross sections at the solar photosphere. In particular, magnetic expansion factor can be defined as

$$
f(\boldsymbol{r}, \Phi)=\frac{\mathcal{S}(\boldsymbol{r}, \Phi)}{\mathcal{S}\left(\boldsymbol{R}_{\odot}, \Phi\right)} \cdot \frac{R_{\odot}^{2}}{r^{2}},
$$

where $\mathcal{S}(\boldsymbol{r}, \Phi)$ is the cross-sectional area of a tube carrying magnetic flux, $\Phi$, at position, $\boldsymbol{r}$, along its axis, $R_{\odot}$, is the solar radius, and $R_{\odot}^{2} / r^{2}$ is a correction factor accounting for the natural expansion of surfaces as $r$ increases in spherical geometry (referred to as "radial expansion" in the text).

From Equation (1) it is possible to define a completely local expansion factor that does not depend on the surface cross section, $\mathcal{S}$. For this we employ "elemental" flux tubes that possess an infinitesimal cross section surrounding a magnetic field line. Let $d \Phi=B d \mathcal{S}$ be an elemental magnetic flux tube, where $B$ is the magnetic field strength along the flux tube axis and $d \mathcal{S}$ is the elemental flux tube cross section. Then, 


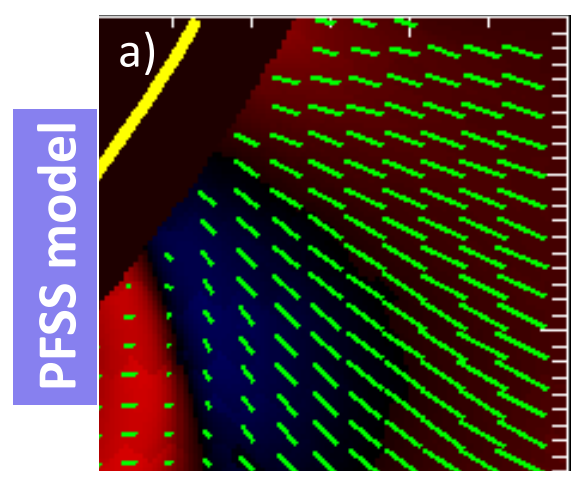

Lin. pol. Azimuth

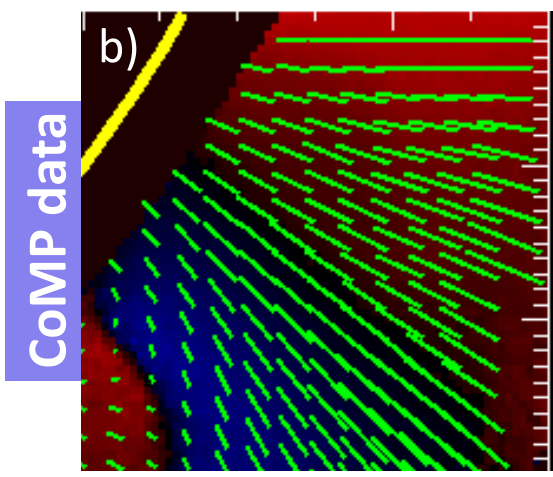

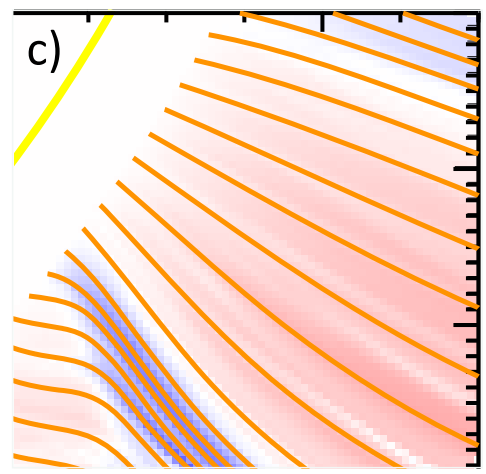

Lin. pol. exp. fac.

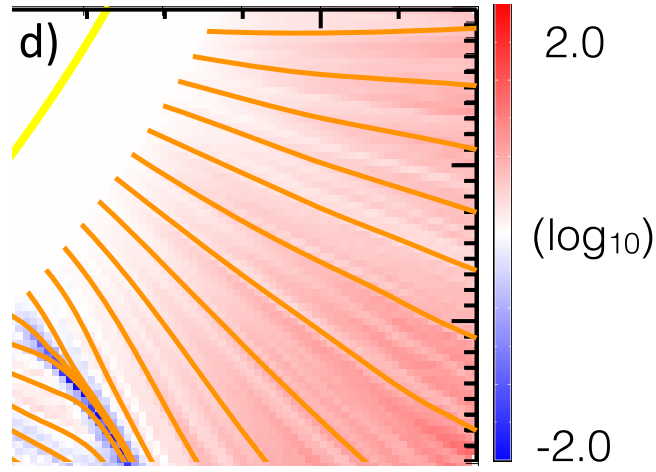

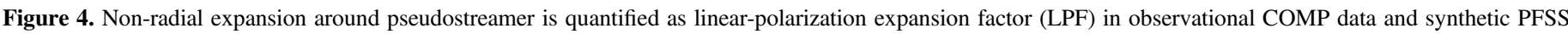

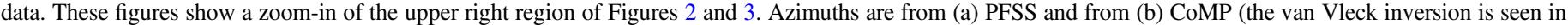

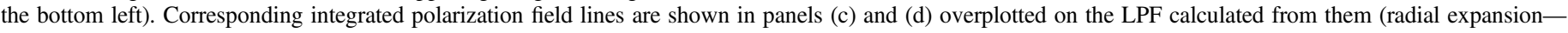
white, super-radial expansion-red, sub-radial expansion-blue; see the Appendix). The solar photosphere is indicated by the yellow curves.

Equation (1) applied to elemental magnetic flux tubes leads to

$$
\begin{aligned}
f(\boldsymbol{r}, d \Phi) & =\frac{d \mathcal{S}(\boldsymbol{r}, d \Phi)}{d \mathcal{S}\left(\boldsymbol{R}_{\odot}, d \Phi\right)} \cdot \frac{R_{\odot}^{2}}{r^{2}}=\frac{(d \Phi / B(\boldsymbol{r}))}{\left(d \Phi / B\left(\boldsymbol{R}_{\odot}\right)\right)} \cdot \frac{R_{\odot}^{2}}{r^{2}}, \\
& =\frac{B\left(\boldsymbol{R}_{\odot}\right)}{B(\boldsymbol{r})} \cdot \frac{R_{\odot}^{2}}{r^{2}}=f(\boldsymbol{r}),
\end{aligned}
$$

where $f(\boldsymbol{r})$ is the local expression of the expansion factor.

When the magnetic field is known in the entire volume, one can compute the local expansion factor from Equation (2) by simply integrating field lines and evaluating the magnetic field strength at each step along the field lines (Wang \& Sheeley 1990). When the magnetic field direction is known, but not the magnetic field strength, we must return to Equation (1), which we will use to define and justify an LPF determined, e.g., from CoMP linear polarization measurements.

\section{A.2. LPF Proxies}

To evaluate the LPF, we build upon methodology developed to compute the squashing factor of magnetic flux tubes (Pariat \& Démoulin 2012; Tassev \& Savcheva 2017). The squashing factor is a measure of the distortion of magnetic flux tubes that provides a means of identifying quasi-separatrix layers (QSLs) in a 3D magnetic field (Demoulin et al. 1996; Titov et al. 2002), and has been extensively used in solar flare analysis to relate photospheric flare ribbons to the topology of the coronal magnetic field (Schmieder et al. 1997; Masson et al. 2009; Savcheva et al. 2012a; Dalmasse et al. 2015). QSLs are regions of strong gradients in connectivity of magnetic field lines and are likely regions for current sheet/layers formation and magnetic reconnection (Aulanier et al. 2005; Janvier et al. 2013).

The method is based on integrating a field line and calculating the field line deviation along it, $\delta \boldsymbol{r}$, from the local variations of the magnetic field vector as obtained from its local interpolation (Longcope \& Strauss 1994; Titov et al. 2003; Tassev \& Savcheva 2017). Once $\delta \boldsymbol{r}$ has been integrated along a field line, we can compute $\delta \boldsymbol{r}_{\perp}$, the component of $\delta \boldsymbol{r}$ perpendicular to the field line. For a 3D flux tube of circular cross section, $\delta \boldsymbol{r}_{\perp}(s)$ effectively represents the diameter of the flux tube at position $\boldsymbol{r}_{s}$ along the flux tube axis. $\delta \boldsymbol{r}_{\perp}(s)$ can thus be used to compute an estimate of the local expansion factor using Equation (1).

Because of the LOS integration effect, the azimuth computed from CoMP only provides an effectively two-dimensional magnetic field direction. Thus, the expansion factor computed from CoMP linear-polarization measurements can only be a proxy of the true, local expansion factor of the coronal magnetic field. Using Equation (1), we define three slightly different proxies of expansion factor

$$
\begin{gathered}
f^{(3 \mathrm{D})}(\boldsymbol{r}(s))=\left(\frac{\left\|\delta \boldsymbol{r}_{\perp}(s)\right\|}{\left\|\delta \boldsymbol{r}_{\perp}\left(s_{\odot}\right)\right\|}\right)^{2} \cdot \frac{R_{\odot}^{2}}{r^{2}}, \\
f^{(2.5 \mathrm{D}, \text { sph. })}(\boldsymbol{r}(s))=\frac{\left\|\delta \boldsymbol{r}_{\perp}(s)\right\| \sin (\theta(s))}{\left\|\delta \boldsymbol{r}_{\perp}\left(s_{\odot}\right)\right\| \sin \left(\theta\left(s_{\odot}\right)\right)} \cdot \frac{R_{\odot}}{r}, \\
f^{(2.5 \mathrm{D}, \text { cyl. })}(\boldsymbol{r}(s))=\frac{\left\|\delta \boldsymbol{r}_{\perp}(s)\right\|}{\left\|\delta \boldsymbol{r}_{\perp}\left(s_{\odot}\right)\right\|} \cdot \frac{R_{\odot}}{r} .
\end{gathered}
$$


Equation (3) assumes that the CoMP data are associated with 3D magnetic flux tubes of the circular cross section, $\mathcal{S}(\boldsymbol{r}(s))=\pi\left(\left\|\delta \boldsymbol{r}_{\perp}(s)\right\| / 2\right)^{2}$, and that axes are in the POS. Equation (4) assumes axisymmetry around the north-south axis of the spherical Sun, in which case the cross section of a flux tube is $\mathcal{S}(\boldsymbol{r}(s))=\left\|\delta \boldsymbol{r}_{\perp}(s)\right\| 2 \pi r(s) \sin (\theta(s))$. Finally, Equation (5) assumes invariance in the direction of the line of sight, meaning that the Sun appears as a cylinder of infinite length, $L$, in the LOS direction; magnetic flux tubes thus have a cross section, $\mathcal{S}(\boldsymbol{r}(s))=\left\|\delta \boldsymbol{r}_{\perp}(s)\right\| L$, and the correction factor that must be applied to remove the natural expansion of surfaces as $r$ increases is now $R_{\odot} / r$. For the case presented in this Letter, we have compared all three LPF proxies and found that although they vary somewhat in magnitude, they are very similar in structure. The conclusions we make comparing the CoMP and PFSS LPF measures are independent of which measure is used; in Figure 4, we show the 3D proxy defined by Equation (3).

\section{References}

Antiochos, S. K., DeVore, C. R., \& Klimchuk, J. A. 1999, ApJ, 510, 485 Antiochos, S. K., Mikic, Z., Titov, V. S., Lionello, R., \& Linker, J. A. 2011, ApJ, 731, 112

Arge, C. N., \& Pizzo, V. J. 2000, JGR, 105, 10465

Ariste, A. L. 2002, ApJ, 575, 87

Arnaud, J., \& Newkirk, G., Jr. 1987, A\&A, 178, 263

Aulanier, G., Pariat, E., \& Demoulin, P. 2005, A\&A, 444, 961

Ba̧k-Stȩślicka, U., Gibson, S. E., Fan, Y., et al. 2013, ApJ, 770, 28

Bąk-Stȩślicka, U., Gibson, S. E., Fan, Y., et al. 2014, in Proc. IAU Symp. 300 Nature of Prominences and their Role in Space Weather, ed. B. Schmieder, J.-M. Malherbe, \& S. T. Wu (Cambridge: Cambridge Univ. Press), 35

Dalmasse, K., Chandra, R., Schmieder, B., \& Aulanier, G. 2015, A\&A, 574, A37

De Rosa, M. L., Schrijver, C. J., Barnes, G., et al. 2009, ApJ, 696, 1780

Del Zanna, G., Aulanier, G., Klein, K.-L., \& Toeroek, T. 2011, A\&A, 526, 137

Demoulin, P., Henoux, J. C., Priest, E. R., \& Mandrini, C. H. 1996, A\&A, 308, 643

Dove, J., Gibson, S., Rachmeler, L. A., Tomczyk, S., \& Judge, P. 2011, ApJL, 731,1
Freeland, S. L., \& Handy, B. N. 1998, SoPh, 182, 497

Gibson, S. 2015, in Solar Prominences, ed. J.-C. Vial \& O. Engvold (Cham: Springer International), 323

Gibson, S., Kucera, T., White, S., et al. 2016, FrASS, 3, 8

Gibson, S. E., Fludra, A., Bagenal, F., et al. 1999, JGR, 104, 9691

Guhathakurta, M., Fludra, A., Gibson, S. E., Biesecker, D., \& Fisher, R. 1999, JGR, 104, 9801

Harvey, J. W. 1969, PhD thesis, Univ. Colorado, Boulder

Harvey, J. W. 2006, in ASP Conf. Ser. 358, Solar Polarization 4, ed. R. Casini \& B. W. Lites (San Francisco, CA: ASP), 419

Hundhausen, A. J. 1972, Coronal Expansion and Solar Wind (Berlin: Springer)

Janvier, M., Aulanier, G., Pariat, E., \& Demoulin, P. 2013, A\&A, 555, A77

Lin, H., \& Casini, R. 2000, ApJ, 542, 528

Lin, H., Kuhn, J. R., \& Coulter, R. 2004, ApJL, 613, 177

Longcope, D. W., \& Strauss, H. R. 1994, ApJ, 437, 851

Lynch, B. J., \& Edmondson, J. K. 2013, ApJ, 764, 87

Masson, S., Pariat, E., Aulanier, G., \& Schrijver, C. J. 2009, ApJ, 700, 559

Newkirk, G., \& Altschuler, M. 1969, BAAS, 1, 288

Pariat, E., \& Démoulin, P. 2012, A\&A, 541, A78

Parnell, C. E., Maclean, R. C., \& Haynes, A. L. 2010, ApJL, 725, 214

Querfeld, C. W., \& Smartt, R. N. 1984, SoPh, 91, 299

Rachmeler, L. A., Gibson, S. E., Dove, J. B., DeVore, C. R., \& Fan, Y. 2013, SoPh, 288, 617

Rachmeler, L. A., Platten, S. J., Bethge, C., Seaton, D. B., \& Yeates, A. R. 2014, ApJL, 787, L3

Riley, P., Lionello, R., Linker, J. A., et al. 2011, SoPh, 274, 361

Sahal-Bréchot, S. 1977, ApJ, 213, 887

Savcheva, A., Van Ballegooijen, A. A., \& DeLuca, E. E. 2012a, ApJ, 744, 78

Schatten, K. H., Wilcox, J. M., \& Ness, N. F. 1969, SoPh, 6, 442

Schmieder, B., Aulanier, G., Demouliun, P., et al. 1997, A\&A, 325, 1213

Tassev, S., \& Savcheva, A. 2017, ApJ, in press

Titov, V. S., Galsgaard, K., \& Neukirch, T. 2003, ApJ, 582, 1172

Titov, V. S., Hornig, G., \& Démoulin, P. 2002, JGR, 107, 1164

Titov, V. S., Mikic, Z., Linker, J. A., Lionello, R., \& Antiochos, S. K. 2011, ApJ, 731, 111

Tomczyk, S., Card, G. L., Darnell, T., et al. 2008, SoPh, 247, 411

Tomczyk, S., Landi, E., Burkepile, J. T., et al. 2016, JGR, 121, 7470

Török, T., Panasenco, O., Titov, V. S., et al. 2011, ApJL, 739, L63

van Vleck, J. H. 1925, PNAS, 11, 612

Wang, Y.-M., Grappin, R., Robbrecht, E., \& Sheeley, N. R. J. 2012, ApJ, 749,182

Wang, Y.-M., \& Sheeley, N. R. J. 1990, ApJ, 355, 726

Wang, Y.-M., Sheeley, N. R. J., \& Rich, N. B. 2007, ApJ, 658, 1340

Zhao, X., \& Webb, D. F. 2003, JGR, 108, 1234 